\title{
A Variant in RUNX3 Is Associated with the Risk of Ankylosing Spondylitis in Koreans
}

\author{
Sung-Min Cho ${ }^{1,2,3}$, Seung-Hyun Jung ${ }^{3,4}$, Yeun-Jun Chung ${ }^{1,2,3 *}$ \\ ${ }^{1}$ Department of Microbiology, College of Medicine, The Catholic University of Korea, Seoul 06591, Korea, \\ ${ }^{2}$ Precision Medicine Research Center, College of Medicine, The Catholic University of Korea, Seoul 06591, Korea, \\ ${ }^{3}$ Integrated Research Center for Genome Polymorphism, College of Medicine, The Catholic University of Korea, Seoul 06591, Korea, \\ ${ }^{4}$ Cancer Evolution Research Center, College of Medicine, The Catholic University of Korea, Seoul 06591, Korea
}

\begin{abstract}
Ankylosing spondylitis (AS) is a chronic autoinflammatory disease that affects the spine and sacroiliac joints. Regarding its etiology, although HLA-B27 is known to be the strongest genetic factor of AS, much evidence suggests the potential contribution of non-MHC genes to the susceptibility to AS. Most of these non-MHC genes have been discovered in non-Asian populations; however, just some of them have been validated in Koreans. In this study, we aimed to identify additional AS-associated single-nucleotide polymorphism (SNP) candidates by replicating the candidate SNPs in Korean AS patients and healthy controls. For this, we selected three SNPs (rs11249215 in RUNX3, rs6556416 in IL12B, and rs8070463 in TBKBP1), which were previously reported as risk factors of AS but have not been studied in Koreans, and performed genotyping assays using a total of 1138 Korean samples (572 AS patients and 566 healthy controls). Of the three SNP candidates, one SNP in RUNX3 (rs11249215) was significantly associated with the risk of AS (odds ratio, 1.31; 95\% confidence interval, 1.02 to 1.68 , $p=0.03$ ). These results will be helpful in elucidating the pathogenesis of AS and may be useful for developing AS risk prediction models in Koreans.
\end{abstract}

Keywords: ankylosing spondylitis, HLA-B27, single nucleotide polymorphism

\section{Introduction}

Ankylosing spondylitis (AS) is a type of chronic autoinflammatory disease that affects the spine and sacroiliac joints. The pathogenesis of AS is not clear; however, it is believed to involve a combination of genetic and environmental factors. Among them, human leukocyte antigen B27 (HLA-B27) is the strongest genetic factor of AS. More than $80 \%$ to $90 \%$ of patients with AS express HLA-B27, and the association between HLA-B27 and AS has been identified in many ethnic groups [1]. However, only a small portion of HLA-B27-positive individuals $(<5 \%)$ develop AS, suggesting a potential contribution of non-MHC genes to the susceptibility to AS [2].

A number of genome-wide association studies (GWASs) of single-nucleotide polymorphisms (SNPs) have identified non-MHC genes associated with AS, such as IL-12B, ERAP1,
RUNX3, IL1R2, and ANTXR2 [3-6]. In addition to SNPs, through genomewide copy number variant (CNV) analysis, CNVs associated with AS have also been identified [7]. These AS-associated non-MHC genes support the contribution of non-MHC genes to the susceptibility to AS. They can also be valuable markers of the development of AS.

From a clinical point of view, the identification of early AS before the appearance of radiological changes in the patient's spine or sacroiliac joint is important, because current treatment options, such as tumor necrosis factor- $\alpha$ (TNF- $\alpha$ ) inhibitors and nonsteroidal anti-inflammatory drugs, after these changes have occurred cannot block or slow down disease progression [8]. Instead, many reports suggest that earlier treatment of TNF- $\alpha$ inhibitors may prevent the progression of AS more effectively [8-10].

In our previous work, to identify AS-associated SNP candidates in Koreans, we selected seven AS-associated SNP candidates, which had been identified in Caucasians by a 
GWAS and consistently replicated in East Asians, and performed a replication study with 285 Korean AS cases and 363 healthy controls [11]. However, in contrast to our expectation, only one of them was replicated in Koreans. In this study, to identify other AS-associated SNP candidates, we selected three SNPs in non-MHC genes from previous GWASs [4, 5, 12, 13] that are associated with AS but have not been tested in Koreans-rs11249215 in RUNX3, rs6556416 in IL12B, and rs8070463 in TBKBP1-and examined them in a large cohort of Koreans with AS and healthy controls.

\section{Methods}

\section{Study subjects}

A total of 1,138 individuals (572 AS patients and 566 healthy controls) were enrolled in this study. All of them were from our previous CNV discovery study [11, 13]. All AS cases were diagnosed according to the Modified New York Criteria [14]. This study was performed with the approval of the Institutional Review Board of the College of Medicine, Catholic University of Korea (CUMC10U170). The patients were aged $26.7 \pm 11.8$ years (85.3\% male and $14.7 \%$ female), and $97.9 \%$ of them were HLA-B27-positive. The controls were aged $23.9 \pm 11.8$ years $(94.7 \%$ male and $5.3 \%$ female), and $2.6 \%$ of them were HLA-B27-positive.

\section{SNP genotyping}

In this study, we selected three SNPs in non-MHC genes from previous GWASs $[4,5,12,13]$ that are associated with AS but have not been tested in Koreans: rs11249215 in RUNX3, rs6556416 in IL12B, and rs8070463 in TBKBP1. SNP genotyping was performed using the ViiA7 system (Life Technologies, Carlsbad, CA, USA) according to the manufacturer's instructions. The thermal cycling program included one cycle at $95^{\circ} \mathrm{C}$ for $10 \mathrm{~min}$, followed by 40 cycles at $95^{\circ} \mathrm{C}$ for $15 \mathrm{~s}$ and $60^{\circ} \mathrm{C}$ for $1 \mathrm{~min}$, and a final extension at $60^{\circ} \mathrm{C}$ for $30 \mathrm{~s}$. Genotypes were determined using ViiA7 software, version 1.2 (Life Technologies).

\section{Statistical analysis}

The differences in allele and genotype frequencies between cases and controls were assessed using chi-squared test and Fisher exact test. Three genetic models (dominant, recessive, and allele) were used to test the SNP associations. Hardy-Weinberg equilibrium had been tested for all three SNPs, and their genotypes were in Hardy-Weinberg equilibrium in our cohort. Statistical analyses were performed using PLINK [15], and p-values less than 0.05 were considered to be significant in all statistical analyses.

\section{Results}

To evaluate the candidate AS-associated genetic markers, we selected three SNPs for a replication study in Koreans (rs11249215, rs6556416, and rs8070463) that have been previously reported as risk factors of AS but have not been studied in Koreans. All of them were located in non-exonic regions: rs11249215 was located in the promoter of RUNX3, rs6556416 was located in the 5'-flanking region of $I L-12 B$, and rs8070463 was located in the promoter of TBKBP1. General information on the three SNPs is summarized in Table 1.

We performed genotyping assays for the three SNPs using a total of 1,138 Koreans (572 AS patients and 566 healthy controls). Minor allele frequencies (MAFs) of the three SNPs in this study $(0.57$ for rs11249215, 0.91 for rs6556416, and 0.44 for rs8070463) were largely similar to those in East Asians from the 1000 Genomes Project, suggesting that our genotyping assays were reliable (Table 1). In the association analysis, one SNP in RUNX3 (rs11249215) was significantly associated with the risk of AS in the recessive model (Table 2). The MAF of rs11249215 was significantly higher in AS patients $(\mathrm{MAF}=0.59)$ than in controls $(\mathrm{MAF}=0.56)$ (odds ratio, 1.31 ; $95 \%$ confidence interval, 1.02 to $1.68 ; \mathrm{p}=0.03$ ). However, significance was not detected in the allelic or dominant model for rs11249215. The other two SNPs in $I L-12 B$ and TBKBP1 showed increased odds ratios, but they

Table 1. General information for the three SNPs

\begin{tabular}{|c|c|c|c|c|c|c|c|c|c|}
\hline \multirow{2}{*}{ SNP } & \multirow{2}{*}{ Genotype } & \multirow{2}{*}{ Position $^{\mathrm{a}}$} & \multirow{2}{*}{ Putative gene } & \multicolumn{6}{|c|}{1000 Genomes Database (MAF) } \\
\hline & & & & All & AFR & AMR & EAS & EUR & SAS \\
\hline rs11249215 & $\mathrm{G} / \mathrm{A}$ & chr1:25297184 & $R U N X 3$ & 0.48 & 0.23 & 0.62 & 0.53 & 0.52 & 0.63 \\
\hline rs6556416 & $\mathrm{A} / \mathrm{C}$ & chr5:158818745 & $I L-12 B$ & 0.83 & 0.88 & 0.81 & 0.91 & 0.70 & 0.84 \\
\hline rs8070463 & $\mathrm{T} / \mathrm{C}$ & chr17:45768836 & TВKBP1 & 0.53 & 0.68 & 0.42 & 0.44 & 0.50 & 0.51 \\
\hline
\end{tabular}

SNP, single-nucleotide polymorphism; Genotype, reference allele/altered allele; MAF, minor allele frequencies; All, average of entire population; AFR, African; AMR, American; EAS, East Asian; EUR, European; SAS, South Asian.

aUCSC GRCh37/hg19. 
Table 2. Association results for the three SNPs

\begin{tabular}{|c|c|c|c|c|c|c|c|c|c|}
\hline \multirow[b]{2}{*}{ SNP } & \multirow[b]{2}{*}{ Risk allele } & \multirow{2}{*}{$\begin{array}{c}\mathrm{AF} \\
\text { (case/control) }\end{array}$} & \multirow{2}{*}{$\begin{array}{c}\text { HoF } \\
\text { (case/control) }\end{array}$} & \multicolumn{2}{|c|}{ Allelic model } & \multicolumn{2}{|c|}{ Recessive model } & \multicolumn{2}{|c|}{ Dominant model } \\
\hline & & & & $\begin{array}{c}\text { OR } \\
(95 \% \mathrm{Cl})\end{array}$ & p-value & $\begin{array}{c}\text { OR } \\
(95 \% \mathrm{Cl})\end{array}$ & p-value & $\begin{array}{c}\text { OR } \\
(95 \% \mathrm{Cl})\end{array}$ & p-value \\
\hline rs11249215 & A & $0.57(0.59 / 0.56)$ & $0.33(0.36 / 0.30)$ & $\begin{array}{c}1.13 \\
(0.96-1.34)\end{array}$ & 0.15 & $\begin{array}{c}1.31 \\
(1.02-1.68)\end{array}$ & 0.03 & $\begin{array}{c}1.00 \\
(0.74-1.36)\end{array}$ & 0.99 \\
\hline rs6556416 & C & $0.91(0.92 / 0.90)$ & $0.83(0.86 / 0.82)$ & $\begin{array}{c}1.33 \\
(0.99-1.78)\end{array}$ & 0.06 & $\begin{array}{c}1.34 \\
\left(0.98^{-1.84)}\right.\end{array}$ & 0.07 & $\begin{array}{c}1.58 \\
(0.52-4.87)\end{array}$ & 0.42 \\
\hline rs8070463 & C & $0.44(0.46 / 0.42)$ & $0.19(0.21 / 0.18)$ & $\begin{array}{c}1.16 \\
\left(0.98^{-1.36)}\right.\end{array}$ & 0.09 & $\begin{array}{c}1.23 \\
(0.91-1.65)\end{array}$ & 0.18 & $\begin{array}{c}1.20 \\
(0.94-1.55)\end{array}$ & 0.15 \\
\hline
\end{tabular}

SNP, single-nucleotide polymorphism; AF, allele frequency; HoF, homozygous risk allele frequencies; OR, odds ratio; Cl, confidence interval.

were not statistically significant (Table 2).

\section{Discussion}

HLA-B27 is the strongest and most well-known genetic factor of AS. However, AS develops in less than $5 \%$ of HLA-B27-positive individuals, suggesting the existence of additional genetic factors. Indeed, a number of genetic markers in non-MHC genes have been identified by SNP GWASs and CNV GWASs [3-7]. In this study, we performed an SNP genotyping assay to evaluate three AS-associated SNP candidates (rs11249215 in RUNX3, rs6556416 in $I L 12 B$, and rs8070463 in TBKBP1) that were discovered in Caucasians but have not been studied in Koreans. To generate more reliable data, we used a large sample set in this study (572 AS patients and 566 healthy controls). To our knowledge, this is the first report of the association of these three SNPs in Koreans. As a result, rs11249215 in RUNX3 was found to be significantly associated with the risk of AS in Koreans. However, rs6556416 in IL12B and rs4389526 in ANTXR2 were not replicated in Koreans.

RUNX3 (Runt-related transcription factor 3) is a member of a family of transcription factors that are important regulators of lineage-specific gene expression. Woolf et al. [16] demonstrated that Runx3 is highly expressed in thymic medulla and that it promotes the differentiation of $\mathrm{T}$ cell to $\mathrm{CD} 8+\mathrm{T}$ cells during thymopoiesis. More recently, RUNX3 was found to be linked to human autoimmune disease and inflammation. For example, Fainaru et al. [17] reported that Runx3 knockout mice develop spontaneous eosinophilic lung inflammation, which is attributed to dendritic cells becoming insensitive to transforming growth factor $\beta$ induced inhibition of maturation. RUNX3 polymorphisms are associated with the susceptibility to autoimmune diseases, such as systemic lupus erythematosus and psoriatic arthritis [18, 19]. Apel et al. [18] identified that RUNX3 is involved in $\mathrm{CD} 8+\mathrm{T}$ lymphocyte differentiation and is related to psoriatic arthritis through a $\mathrm{T}$ cell-mediated mechanism. The association between the RUNX3 polymorphism rs11249215 and AS has also been reported in Caucasian [4] and Han Chinese populations [12]. Furthermore, Vecellio et al. [20] demonstrated that rs4648889 in RUNX3 is associated with AS and that its risk allele reduces RUNX3 expression. Consistent with previous studies, in this study, we confirmed that rs11249215 in RUNX3 is significantly associated with the risk of AS in Koreans in the recessive model. Because the homozygous risk allele frequency is important when an SNP is a recessive marker, we examined the homozygous risk allele frequency for rs11249215 and found that it was significantly higher in AS patients (homozygous risk allele frequencies $[\mathrm{HoF}]=0.36$ ) than in controls ( $\mathrm{HoF}=0.33$ ) (Table 2 ). Of note, the MAF of rs11249215 in controls was largely similar to that in East Asians from the 1000 Genomes Project, suggesting that our study group was not biased and that the genotyping was appropriate. Because rs11249215 is located in the promoter of RUNX3, further studies on its molecular effects will help elucidate the pathogenesis of AS. In addition, considering that new SNPs are being reported to be associated with AS, other replication studies of newly identified SNPs are also needed.

In conclusion, we performed a replication study of three SNPs with a relatively large cohort of samples and found that rs11249215 in RUNX3 is significantly associated with the risk of AS in Koreans. These results will be helpful in elucidating the pathogenesis of AS and may be useful for developing risk prediction models for AS in Koreans.

\section{Acknowledgments}

This study was supported by a grant from the Korean Health Technology R\&D Project, Ministry for Health and Welfare, Republic of Korea (HI14C3417). 


\section{References}

1. van der Linden SM, Valkenburg HA, de Jongh BM, Cats A. The risk of developing ankylosing spondylitis in HLA-B27 positive individuals: a comparison of relatives of spondylitis patients with the general population. Arthritis Rheum 1984;27:241-249.

2. Brophy S, Hickey S, Menon A, Taylor G, Bradbury L, Hamersma J, et al. Concordance of disease severity among family members with ankylosing spondylitis? J Rheumatol 2004;31:1775-1778.

3. Wellcome Trust Case Control Consortium; Australo-AngloAmerican Spondylitis Consortium (TASC); Burton PR, Clayton DG, Cardon LR, Craddock N, et al. Association scan of 14,500 nonsynonymous SNPs in four diseases identifies autoimmunity variants. Nat Genet 2007;39:1329-1337.

4. Evans DM, Spencer CC, Pointon JJ, Su Z, Harvey D, Kochan G, et al. Interaction between ERAP1 and HLA-B27 in ankylosing spondylitis implicates peptide handling in the mechanism for HLA-B27 in disease susceptibility. Nat Genet 2011;43:761-767.

5. Lin Z, Bei JX, Shen M, Li Q, Liao Z, Zhang Y, et al. A genome-wide association study in Han Chinese identifies new susceptibility loci for ankylosing spondylitis. Nat Genet 2011;44:73-77.

6. Australo-Anglo-American Spondyloarthritis Consortium (TASC), Reveille JD, Sims AM, Danoy P, Evans DM, Leo P, et al. Genome-wide association study of ankylosing spondylitis identifies non-MHC susceptibility loci. Nat Genet 2010;42: 123-127.

7. Jung SH, Yim SH, Hu HJ, Lee KH, Lee JH, Sheen DH, et al. Genome-wide copy number variation analysis identifies deletion variants associated with ankylosing spondylitis. Arthritis Rheumatol 2014;66:2103-2112.

8. Haroon N, Inman RD, Learch TJ, Weisman MH, Lee M, Rahbar MH, et al. The impact of tumor necrosis factor alpha inhibitors on radiographic progression in ankylosing spondylitis. Arthritis Rheum 2013;65:2645-2654.

9. Sieper J, Rudwaleit M. How early should ankylosing spondylitis be treated with tumour necrosis factor blockers? Ann Rheum Dis 2005;64 Suppl 4:iv61-iv64.

10. Wallis D, Inman RD. Recognition of preclinical and early disease in axial spondyloarthritis. Rheum Dis Clin North Am 2014;40:685-697.

11. Jung SH, Cho SM, Yim SH, Kim SH, Park HC, Cho ML, et al.
Developing a risk-scoring model for ankylosing spondylitis based on a combination of HLA-B27, single-nucleotide polymorphism, and copy number variant markers. J Rheumatol 2016;43:2136-2141.

12. Lian Z, Chai W, Shi LL, Chen C, Liu J, Wang Y. Analysis of $P P A R G C 1 B, R U N X 3$ and TBKBP1 polymorphisms in Chinese Han patients with ankylosing spondylitis: a case-control study. PLoS One 2013;8:e61527.

13. Zhang L, Fan D, Liu L, Yang T, Ding N, Hu Y, et al. Association study of IL-12B polymorphisms susceptibility with ankylosing spondylitis in mainland Han population. PLoS One 2015;10: e0130982.

14. van der Linden S, Valkenburg HA, Cats A. Evaluation of diagnostic criteria for ankylosing spondylitis. A proposal for modification of the New York criteria. Arthritis Rheum 1984;27: 361-368.

15. Purcell S, Neale B, Todd-Brown K, Thomas L, Ferreira MA, Bender D, et al. PLINK: a tool set for whole-genome association and population-based linkage analyses. Am J Hum Genet 2007;81:559-575.

16. Woolf E, Xiao C, Fainaru O, Lotem J, Rosen D, Negreanu V, et al. Runx3 and Runx1 are required for CD8 T cell development during thymopoiesis. Proc Natl Acad Sci U S A 2003;100: 7731-7736.

17. Fainaru O, Woolf E, Lotem J, Yarmus M, Brenner O, Goldenberg D, et al. Runx3 regulates mouse TGF-beta-mediated dendritic cell function and its absence results in airway inflammation. EMBO J 2004;23:969-979.

18. Apel M, Uebe S, Bowes J, Giardina E, Korendowych E, Juneblad K, et al. Variants in RUNX3 contribute to susceptibility to psoriatic arthritis, exhibiting further common ground with ankylosing spondylitis. Arthritis Rheum 2013;65: 1224-1231.

19. Wang W, Ma Y, Xu Y, Sheng Y, Gao J, Zuo X, et al. RUNX3 gene polymorphisms are associated with clinical features of systemic lupus erythematosus in Chinese Han population. $J$ Dermatol Sci 2015;80:69-71.

20. Vecellio M, Roberts AR, Cohen CJ, Cortes A, Knight JC, Bowness $\mathrm{P}$, et al. The genetic association of RUNX3 with ankylosing spondylitis can be explained by allele-specific effects on IRF4 recruitment that alter gene expression. Ann Rheum Dis 2016;75:1534-1540. 